\title{
Comparison of Body Fat Results from 4 Bioelectrical Impedance Analysis Devices vs. Air Displacement Plethysmography in American Adolescent Wrestlers
}

\author{
Melissa M. Montgomery ${ }^{1}$, Risto H. Marttinen², Andrew J. Galpin ${ }^{1}$ \\ ${ }^{1}$ Center for Sport Performance, Department of Kinesiology, California State University, Fullerton, 800 N. State College Blvd., Fullerton, \\ CA 92831 USA, ${ }^{2}$ Department of Kinesiology, California State University, Fullerton, 800 N. State College Blvd., Fullerton, CA 92831 \\ USA
}

Corresponding Author: Andrew J. Galpin, E-mail: agalpin@fullerton.edu

\section{ARTICLE INFO}

Article history

Received: August 01, 2017

Accepted: September 25, 2017

Published: October 31, 2017

Volume: 5 Issue: 4

Conflicts of interest: None

Funding: The research was funded by the National Wrestling Coaches Association (USA) and the National Federation of State High School Associations (USA).

\begin{abstract}
Background: Accurate and accessible methods of body composition are necessary to ensure health and safety of wrestlers during competition. The most valid and reliable instruments are expensive and relatively inaccessible to high school wrestlers; therefore, more practical technology is needed. Objective: To compare body fat percentage (BF\%) results from 4 bioelectrical impedance analysis (BIA) devices to those from air displacement plethysmography (ADP) in adolescent wrestlers. Methodology: 134 adolescent male and female wrestlers $(1.72 \pm 0.9 \mathrm{~m}$, $66.8 \pm 14.3 \mathrm{~kg}, 15.6 \pm 1.1 \mathrm{yrs}$.) were tested for hydration and then completed 4 body composition tests with different BIA devices and one with Bod Pod. Relative and absolute agreement were assessed between each BIA device and ADP on a single day. Results: When compared with ADP, all devices demonstrated excellent reliability (ICC $(2,1)$ ) range: 0.88-0.94), but questionable measurement error (SEM range: 2.3-3.6 \%BF). Bland-Altman plots revealed that each bioelectrical impedance device we tested over-estimated body fat percent in high school wrestlers (range: $0.8-3.6 \% \mathrm{BF}$ ) and demonstrated wide 95\% limits of agreement (range: 15.0 $20.8 \% \mathrm{BF}$ ) compared to ADP. Conclusions: The devices investigated demonstrated reasonable measurement accuracy. However, wide margins of error of each device were noted. Caution should be taken when assessing adolescent wrestlers with lower amounts of body fat, as it may result in failing to identify those who do not meet the minimum body fat percentage for competition. The governing bodies should use the research data in the decision-making process regarding appropriate devices for use in their weight management programs.
\end{abstract}

Key words: Body Composition, Wrestling, Bioelectrical Impedance, Air Displacement Plethysmography

\section{INTRODUCTION}

Because of the danger associated with the unsafe weight loss practices of wrestlers, weight certification programs, such as that developed by the National Wrestling Coaches' Association (NWCA; www.nwca.org) require body composition assessments at the beginning of the season in order to determine the lowest weight class in which each wrestler can safely compete. In the United States, this is determined based on the wrestler's body fat percentage. Currently, the lowest percent body fat at which a wrestler is permitted to compete is $7 \%$ for boys and $12 \%$ for girls (National Federation of State High School Associations, 2016b). Accordingly, accurate and accessible methods of body composition are necessary to perform these important assessments. Dual energy x-ray absorptiometry (DXA) and air displacement plethysmography (ADP) have been shown to be reliable and valid tools for assessing body composition in adults (Andre- oli, Scalzo, Masala, Tarantino, \& Guglielmi, 2009; Fields, Goran, \& McCrory, 2002) and adolescents (Fields et al., 2002), but these instruments are expensive and not portable; hence, they are relatively inaccessible to wrestlers. Further, they are impractical for assessing large numbers of wrestlers. Skinfolds are also a considered valid method for predicting body fat; however, the validity of this method relies upon the skill of highly-trained assessors. As such, practical technologies are needed for the purpose of accurately assessing body composition (Utter et al., 2005).

Bioelectrical impedance analysis (BIA) has been adopted by some wrestling governing bodies as a surrogate for the aforementioned methods because of its greater accessibility due to lower price, increased portability, ease of use, and smaller risk of user error compared to other methods. (Utter \& Lambeth, 2010) One single frequency leg-to-leg BIA device (Tanita TBF-300WA; Tanita Corporation of America, Inc., Arlington Heights, IL, USA) is currently approved for

Published by Australian International Academic Centre PTY.LTD.

Copyright (c) the author(s). This is an open access article under CC BY license (https://creativecommons.org/licenses/by/4.0/) http://dx.doi.org/10.7575/aiac.ijkss.v.5n.4p.18 
pre-season assessment of body composition and determination of the minimum wrestling weight by approximately 27 state high school federations in the United States (NFHS. org). This device was approved because it demonstrated acceptable agreement with hydrostatic weighing when measuring fat-free mass in high school wrestlers (Utter et al., 2005). More recently, a multi-frequency BIA device was reported to have a strong correlation and no difference when compared to hydrostatic weighing in high school wrestlers. (Utter et al., 2005) However, the researchers also reported a proportional bias: the BIA device overestimated FFM of wrestlers in the lighter weight classes and underestimated FFM of those in higher weight classes.

With the advances in technology and differences in the design (frequency, electrodes, points of contact, etc.) and proprietary body composition prediction algorithms between manufacturers, one must interpret the findings from individual devices cautiously as the aforementioned factors likely influence our interpretations of accuracy if attempting to extrapolate to new BIA devices. Accordingly, instruments using the most current technology should be investigated in order to determine which instruments are acceptable for assessing body composition, thus ensuring safety for competition. Therefore, the purpose of this study is to compare the body fat percent $(\mathrm{BF} \%)$ results from 4 BIA devices vs. that from ADP in American high school wrestlers, aged 1418 years. We chose ADP as the reference because it accepted as a valid method for measuring whole body composition, is not prone to tester error, and is also approved in most states as a final authority for body composition assessment in the case that a wrestler appeals their initial body composition assessment via some other method.

\section{METHODOLOGY}

\section{Participants}

112 male and 22 female wrestlers from local high schools participated in this study. The proportion of females included in this study (18\%) was chosen to ensure that the proportion of secondary school female wrestlers in the United States ( $\sim 5 \%$ nationally (National Federation of State High School Associations, 2016a)) was represented. To be included in the study, they had to be currently competing on their school wrestling team, euhydrated (urine specific gravity $<1.025$ ), and had not exercised within 4 hours nor eaten within 3 hours prior to testing. Assent and consent were provided by the participant and parent/guardian, respectively, according to the university Institutional Review Board protocol.

\section{Procedures}

Testing took place during the morning hours. Upon arrival, the wrestler was asked to confirm eligibility for participation before being enrolled and provided a subject code. They were then measured for body height with a wall-mounted digital stadiometer (Model DHRWM; Detecto; Webb City, $M O, U S A)$. Wrestlers were measured in bare feet and were asked to "stand as tall and still as possible with their feet completely on the floor". This measurement was used for all subsequent body composition tests. Because of the known influence of hydration on body composition assessment devices, all wrestlers were asked to provide a urine sample. Urine specific gravity (USG) was immediately measured with a digital handheld "pen" refractometer (Atago USA, Inc.; Bellevue, WA, USA). The wrestler was disqualified from testing if the USG was above 1.025 (Armstrong et al., 1994; California Interscholastic Federation, 2016).

\section{Body composition testing}

Properly hydrated wrestlers then completed body composition testing in a randomized order. Because of the known sensitivity of the BIA devices to fluid pooled in the distal extremity (Lozano-Nieto \& Turner, 2001), if the ADP measurement was completed before any BIA measurements, investigators ensured the wrestler remained in a standing position for at least 10 minutes before any BIA testing was performed. All participants wore minimal clothing for all testing. For boys, this included: compression shorts, boxer briefs, or a wrestling singlet. For girls, acceptable apparel included: compression shorts and a sports bra, or a singlet. All wrestlers wore the same clothing for each body composition test.

\section{Air displacement plethysmography (ADP) testing}

ADP was tested with the Bod Pod (COSMED USA, Inc., Concord, CA, USA). (McCrory, Gomez, Bernauer, \& Mole, 1995) The manufacturer's complete quality control procedures were performed at the beginning of each testing day. The manufacturer's protocol was followed for each participant, which included approximately 3 minutes of setup and calibration and 2 minutes of actual testing. Briefly, the wrestler's demographic information was input into the software (version 5.4.1), which then prompted the researchers to calibrate the device and scale, weigh the wrestler on the Bod Pod scale, and then perform the ADP measurement. The Brozek equation (Brozek, Grande, Anderson, \& Keys, 1963) and predicted thoracic gas volume were used to predict percent body fat from body volume, respectively. The participant was asked to don a swim cap to compress their hair before entering the Bod Pod. They were then instructed to sit still and breathe normally for the duration of the test. The software automatically completed two measurements, with a third in the event that the $1^{\text {st }}$ and $2^{\text {nd }}$ measurements disagreed by more than $3 \%$. Percent body fat was automatically calculated by the software.

\section{Bioelectrical impedance analysis testing}

Participants completed body composition assessment with 4 BIA devices: Accuniq BC310 (ACC) (Accuniq Co., Ltd., Seoul, KOR), InBody 120 (IB120) (InBody USA, Cerritos, CA, USA), InBody 270 (IB270) (InBody USA, Cerritos, CA, USA), and the Tanita TBF-300WA plus (TAN) (Tanita Corporation of America, Inc., Arlington Heights, IL, USA). Specifications for each device are listed in Table 1. 
Before the BIA tests, the participants wiped the palms of their hands and the soles of their feet with wipes soaked with an antibacterial solution $(0.9 \%$ Sodium Chloride, $15 \mathrm{ppm}$ Isothiazolinone and 150ppm Didecyldimethylammonium chloride) (InBody Tissue; InBody US, Cerritos, CA, USA) in order to ensure clean contacts with the devices and to enhance electrical conductivity. Participants then followed the prompts from each device to complete testing. In brief, these prompts asked the wrestler to step onto the scale and stand still while their body mass was measured. Then the investigators were prompted to enter the wrestler's sex, age/date of birth and height. The wrestler was then asked to align their heels and forefeet with the electrodes on the measurement scale and ensure maximum contact area. For the tetrapolar devices (IB120, IB270, and ACC), the wrestler was also asked to align their thumbs, fingers, and palms to maximize contact area with the electrodes while holding onto the device handles. They were then instructed to extend their elbows and slightly abduct their shoulders to ensure that their arms were not touching their torso. The TAN device was the only bipolar device used and as such, did not have handles. For this test, the wrestler only had to align their feet on the 4 electrodes (heels and forefeet). Once the proper positioning was achieved for each device, the wrestler was then asked to stand still and remain silent while the device completed the body composition measurement, which took 30 seconds on average. The investigators administered and supervised the entire test to ensure that the wrestler maintained the proper position and did not move.

All five body composition assessments were completed within 90 minutes. During that time, the wrestlers were not allowed to eat, drink, or perform physical activity. They were encouraged to empty their bladder if the need arose during the testing appointment. None of the wrestlers needed to urinate during the testing session.

\section{Data Reduction and Statistical Analysis}

The percent body fat $(\% \mathrm{BF})$ automatically calculated by each device was used for analysis. Intraclass correlation (ICC $(2,1))$ and standard error of measurement (SEM) were calculated to assess relative agreement. Bland-Altman plots (Bland \& Altman, 1986) were created to visually assess the absolute agreement and precision between each device and the Bod Pod. To construct the plots, the difference between each device and Bod Pod was determined by subtracting the $\mathrm{BF} \%$ value acquired from each device from that acquired from the Bod Pod. Then, the average of the value from the Bod Pod and each BIA device $((\mathrm{BIA}+\mathrm{BP}) / 2)$ was calculated. Each individual's data were then plotted with the average value measurements (MEAN \%BF) on the $\mathrm{X}$-axis and the difference between the two devices (DIFF $\% \mathrm{BF}$ ) on the Y-axis to allow for visualization of the relationship between the difference and the mean. The new MEAN and DIFF variables were inspected and confirmed to conform to a normal distribution. As such, the $95 \%$ limits of agreement $( \pm 1.96 \times \mathrm{SD})$ were calculated and displayed on each plot as a metric of precision along with the mean difference (bias).

One sample t-tests were first used to determine whether fixed bias (bias is statistically different from zero) existed for the devices. Then, in order to determine whether a proportional bias might be present, the difference in $\mathrm{BF} \%$ between each device and ADP were regressed on the bias of each device to visually display their relation to one another. In the case that the difference between the BIA and ADP instrument was dependent on the magnitude of $\mathrm{BF} \%$, the $95 \% \mathrm{lim}$ its of agreement was recalculated using a regression-based approach. (Bland \& Altman, 1999)

All analyses were performed with IBM SPSS version 24 (IBM Corp., Armonk, NY, USA). A significance level of $<0.05$ was determined a priori.

\section{RESULTS}

112 male $(1.72 \pm 8.4 \mathrm{~m} ; 67.9 \pm 15.1 \mathrm{~kg} ; 15.6 \pm 1.1 \mathrm{yrs} . ; 23.0 \pm 4.8$ BMI; 14.9 $\pm 7.7 \%$ body fat) and 22 female $(1.59 \pm 6.0 \mathrm{~m}$; $61.1 \pm 7.7 \mathrm{~kg} ; 15.5 \pm 1.1$ yrs.; $24.2 \pm 3.3 \mathrm{BMI} ; 24.2 \pm 3.3 \%$ body fat) wrestlers participated in this study. For descriptive purposes, wrestlers were classified by the 2017 age division and weight classes (USA Wrestling, 2016) (Figure 1). Descrip-

Table 1. Specifications for Bioelectrical Impedance Analysis (BIA) devices

\begin{tabular}{lllll}
\hline Device & Accuniq BC310 & InBody 120 & InBody 270 & Tanita TBF-300WA plus \\
\hline Frequency & Single & Multiple & Multiple & Single \\
& $(50 \mathrm{~Hz})$ & $(20 \& 100 \mathrm{kHz})$ & $(20 \& 100 \mathrm{kHz})$ & $(50 \mathrm{~Hz})$ \\
Electrode & Tetrapolar, & Tetrapolar, & Tetrapolar, & Bipolar, \\
method & 8 electrodes & 8 electrodes & 8 electrodes & 4 electrodes
\end{tabular}
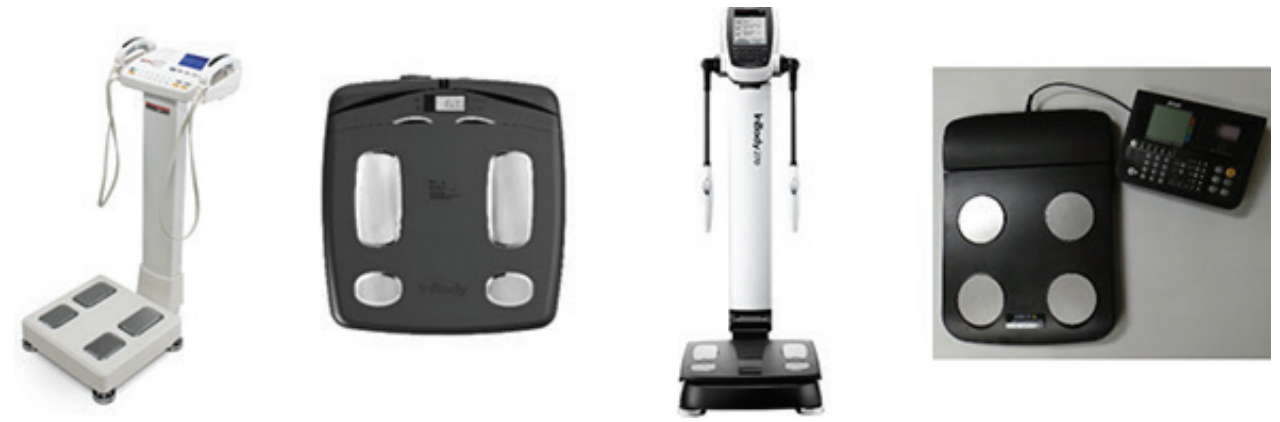
tives for the body fat percentage results from each device are displayed in Table 2.

\section{Intraclass Correlations}

The intraclass correlations (Table 3) can be interpreted as the frequency with which the two devices agree within the bounds of the standard error of the measurement (SEM). For example, the results indicate that the $\% \mathrm{BF}$ results from the IB120 are within $2.3 \%$ of the Bod Pod results, $94 \%$ of the time. When qualitatively comparing the ICC's, the IB120 and IB270 demonstrated slightly better agreement and precision, compared to the other two devices. However, the ICC for the Tanita device still achieved what is commonly considered as "excellent" agreement, with slightly less precision. While the Accuniq device's ICC was not appreciably worse, the SEM is larger, indicating its precision is about $1 \%$ lower than the other devices.

\section{Fixed Bias: One sample T-tests}

The t-tests revealed that the bias between each BIA device and ADP was significant (null hypothesis: bias=0) (Table 4). This bias was smallest for the IB120 device, which overestimated body fat by $0.8 \%$ and largest for the ACC which overestimated by $3.6 \%$ body fat. However, as noted in Table 2 , the effect sizes for the mean difference between each BIA device and the Bod Pod were small (0.08-0.38).

\section{Proportional Bias: Bland-Altman plots and Regression analysis}

In order to determine whether the measurement error was uniform across the range of lean body mass values, the Bland-Altman plots were first visually inspected (Figure 3). Then, linear regressions determined whether the mean BF\% was a significant predictor of the bias of each instrument. Upon inspection of the plots (Figure 2), outliers were detected for each device, which is typical. In addition to the fixed bias noted, wide limits of agreement, spanning 15.7$20.8 \%$ were present for all devices as well (Table 5). In other words, for the IB120, on average we expect about $1 \%$ over-estimation of body fat, but we can still reasonably expect anywhere from $8.6 \%$ over-estimation to a $7 \%$ under-estimation.

The regression analysis revealed no relationship between the difference in $\mathrm{BF} \%$ and the mean $\mathrm{BF} \%$ between devices for the IB120 $\left(\mathrm{F}_{1,132}=3.80 ; \mathrm{P}=0.05\right)$ or the TAN $\left(\mathrm{F}_{1,132}=0.26\right.$; $\mathrm{P}=0.62)$. However, these relationships were significant for the ACC $\left(\mathrm{F}_{1,133}=26.43 ; \mathrm{P}<0.001\right)$ and the IB270 $\left(\mathrm{F}_{1,132}=8.66\right.$; $\mathrm{P}=0.004)$. Accordingly, new regression-based $95 \%$ LOA's were calculated for these two devices (Figure 2A and 2C).

Table 2. Descriptives for body fat percentage results (mean \pm SD) for Bod Pod and each bioelectrical impedance analysis device. Mean difference (device-Bod Pod), 95\% confidence intervals and effect sizes are provided

\begin{tabular}{lcccc}
\hline Instrument & Mean \pm SD & $\begin{array}{c}\text { Mean difference } \dagger \text { and range } \\
(\text { Min-Max) }\end{array}$ & $\mathbf{9 5 \% ~ C I ~}$ & Effect size \\
\hline Bod Pod & $16.7 \pm 8.4$ & -- & -- & -- \\
Accuniq BC310 & $20.3 \pm 10.5$ & $-3.6(-16.4-14.4)$ & $0.12-1.49$ & 0.38 \\
InBody 120 & $17.4 \pm 9.1$ & $-0.81(-13.6-11.0)$ & $1.83-3.13$ & 0.08 \\
InBody 270 & $19.2 \pm 9.4$ & $-2.5(-15.6-7.1)$ & $1.38-2.98$ & 0.28 \\
Tanita TBF-300WA plus & $18.9 \pm 8.3$ & $-2.2(-3.1-13.3)$ & $2.66-4.47$ & 0.26 \\
\hline
\end{tabular}

$\uparrow$ Negative number indicates overestimation by instrument when compared to air displacement plethysmography

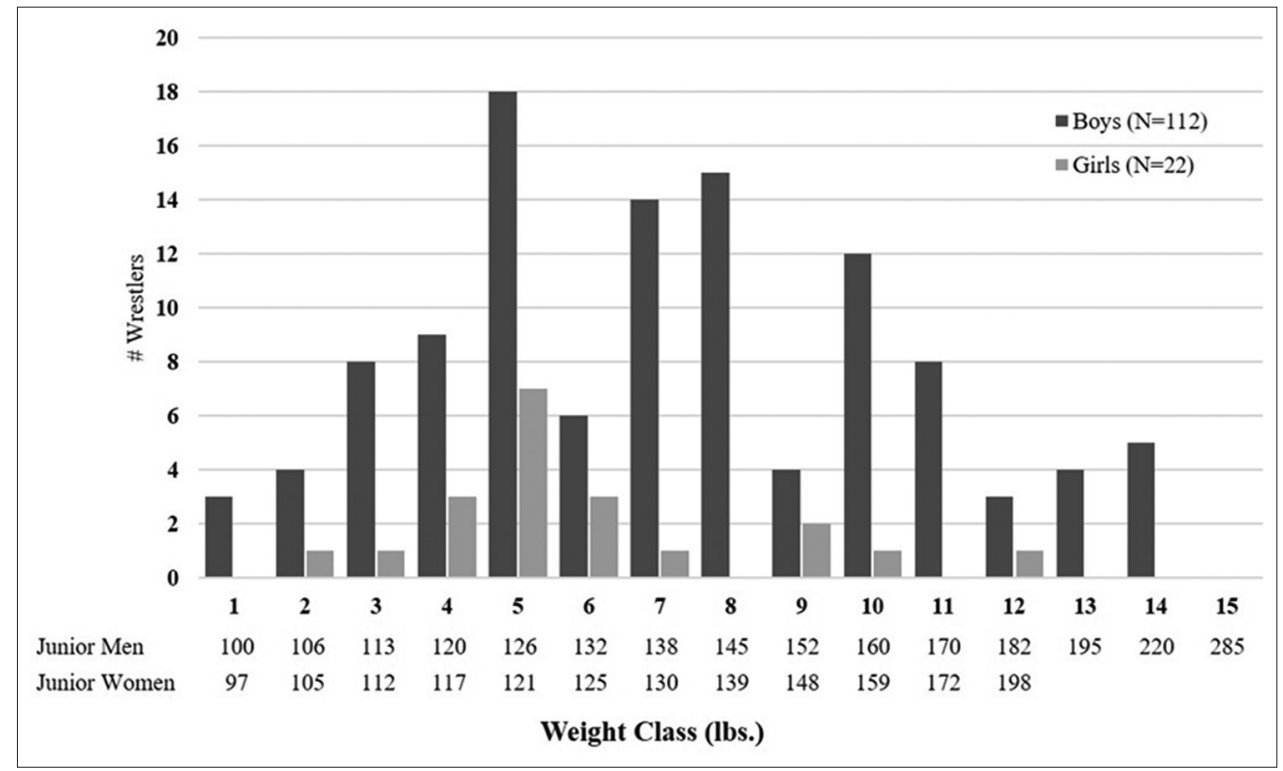

Figure 1: Distribution of wrestlers across weight classes (lbs.) 


\section{DISCUSSION}

We compared the body fat measurements from 4 bioelectrical impedance devices against results from air displacement plethysmography. Our primary finding was that each of the BIA devices produced significantly higher body fat percentage results than the Bod Pod, which ranged from overestimation of $0.8 \%$ to $3.6 \%$. However, despite the statistical significance of the differences, the effect sizes were small. The wide limits of agreement may be somewhat problematic in wrestlers at the lower end of the body fat spectrum, for example when approaching the National Federation of High Schools Association's minimum $7 \%$ and $12 \%$ cut-offs for boys and girls, respectively.

While several previous studies have evaluated the agreement between BIA devices and a reference standard (e.g. hy-

Table 3. Intraclass correlations (ICC 2,1) and SEM for relative agreement of percent body fat $(\% \mathrm{BF})$ results between each device and the Bod Pod

\begin{tabular}{lcc}
\hline Instrument & ICC (2,1) & $\begin{array}{c}\text { SEM } \\
(\mathbf{\%} \text { BF })\end{array}$ \\
\hline Bod Pod & -- & -- \\
Accuniq BC310 & 0.88 & 3.62 \\
InBody 120 & 0.94 & 2.30 \\
InBody 270 & 0.93 & 2.43 \\
Tanita TBF-300WA plus & 0.90 & 2.67 \\
\hline
\end{tabular}

drostatic weighing, dual energy x-ray, or air displacement plethysmography), direct comparisons are difficult due to differences in BIA devices and changes in BIA technology (e.g., electrode type, prediction algorithms, etc.), as well as varying methods of assessing agreement (e.g. t-tests, ICC's, correlations, and Bland-Altman plots). Of the previous work in the wrestling population, two studies have reported no difference in the fat-free mass results between single- and multi-frequency BIA devices and hydrostatic weighing in high school (Utter et al., 2005) and college wrestlers (Utter \& Lambeth, 2010), respectively. However, a proportional bias was also noted in both datasets, indicating that the BIA devices tended to over-estimate fat-free mass in the lighter individuals, while under-estimating in the heavier wrestlers. Because we compared $\mathrm{BF} \%$ from the BIA devices in the current study to the ADP reference, our results cannot be directly compared to the previous studies. However, as an illustration of the relative effects of differences in $\mathrm{BF} \%$ on fat-free mass, Table 6 displays the expected results from each device based on a $150 \mathrm{lb}$. wrestler with $12 \%$ body fat, as assessed by the Bod Pod according to the fat-free mass calculation used in the NCAA Weight Management Program (National Collegiate Athetics Association, 2016) to calculate the lowest allowable weight for competition.

We observed high intraclass correlations for each device, indicating that a measurement from a device would agree with the Bod Pod more than $90 \%$ of the time (albeit within an
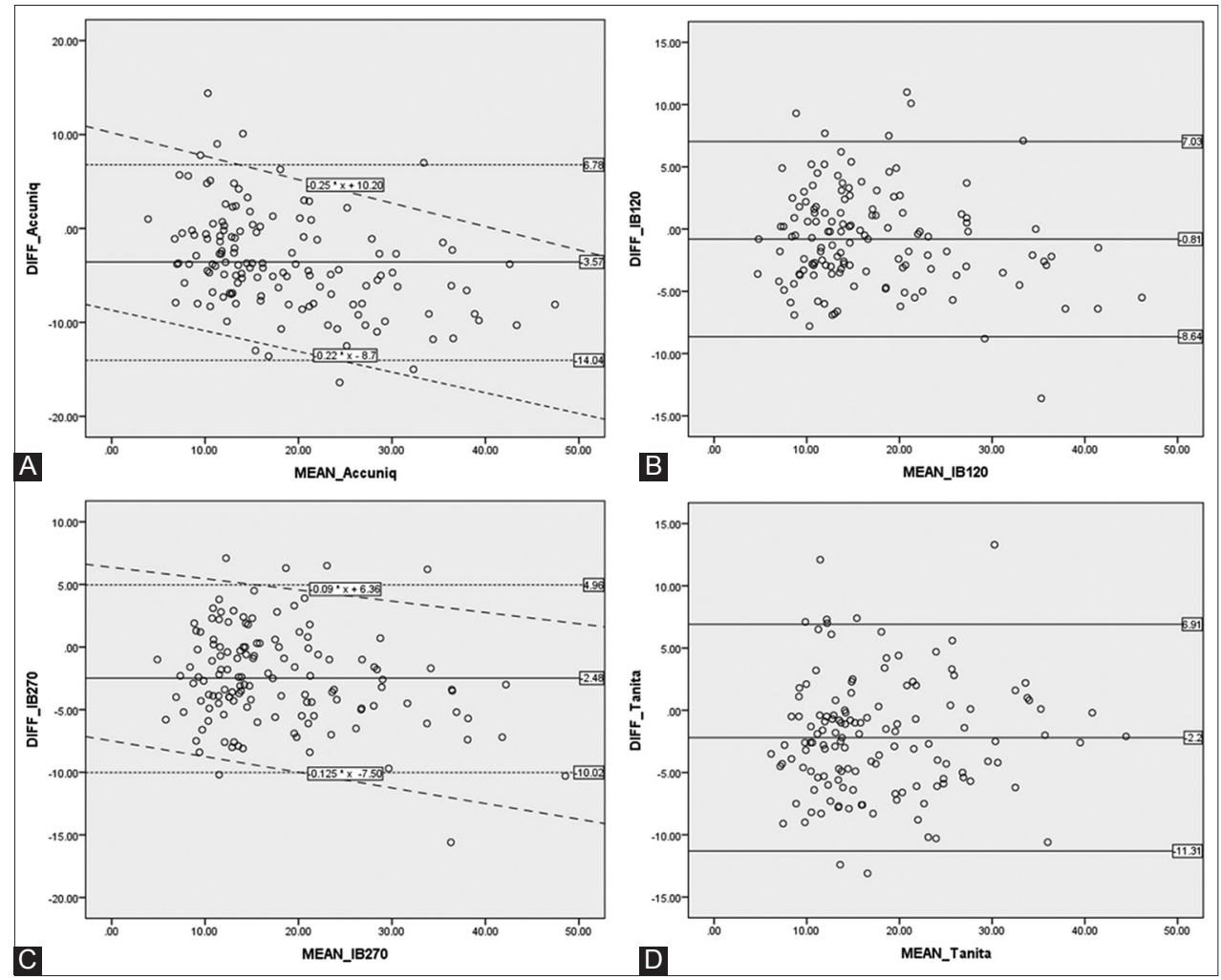

Figure 2: Bland-Altman plots visualize the relationship between the difference in percent body fat result between the Bod Pod and each device (DIFF) and the average percent body fat between the Bod Pod and each device (MEAN): A) Accuniq BC310, B) InBody 120, C) InBody 270, D) Tanita TBF-300WA plus. Solid horizontal line represents the bias (average DIFF). The dotted lines represent the upper and lower boundaries of the $95 \%$ limits of agreement $(1.96 \pm \mathrm{SD}$ of DIFF). The diagonal dashed lines represent the revised regressionbased $95 \%$ limits of agreement calculated due to the presence of proportional bias 
Table 4. One sample t-test results for bias in percent body fat (\%BF; Bod Pod - device) calculated by each device

\begin{tabular}{lcccc}
\hline Instrument & Bias (\%BF) & t & P-value & 95\% CI \\
\hline Accuniq BC310 & -3.63 & -7.91 & 0.00 & $-4.54--2.72$ \\
InBody 120 & -0.81 & -2.33 & 0.02 & $-1.49--0.12$ \\
InBody 270 & -2.53 & -7.66 & 0.00 & $-3.18--1.88$ \\
Tanita TBF-300WA & -2.20 & -5.48 & 0.00 & $-2.99--1.40$ \\
\hline
\end{tabular}

Table 5. Bias and 95\% limits of agreement for each device

\begin{tabular}{lcc}
\hline Instrument & Bias (\%) & $\begin{array}{c}\text { 95\% LOA } \\
\text { (range \%) }\end{array}$ \\
\hline Accuniq BC310 & -3.63 & $-14.04-6.78(20.8 \%)$ \\
InBody 120 & -0.81 & $-8.64-7.03(15.7 \%)$ \\
InBody 270 & -2.53 & $-10.02-4.96(15.0 \%)$ \\
Tanita TBF-300WA & -2.20 & $-11.31-6.91(18.2 \%)$ \\
\hline
\end{tabular}

approximate $2.5 \%$ body fat margin of error). Yet, that there were significant differences in the relative agreement (bias) between each device and the Bod Pod provides an illustration of the shortcomings of only analyzing the absolute agreement via ICC's as described by Altman and Bland (Bland \& Altman, 1986). The relative agreement (bias) we observed for the devices studied are in accordance with Lee et al (Lee et al., 2017), who reported a $3 \%$ and $4.5 \%$ bias in school-aged (7-12 years old) boys and girls, respectively, when comparing the InBody 230 multiple frequency BIA device (similar to the IB270 device used in this study) with DXA. An additional study (Utter \& Lambeth, 2010) compared fat-free mass measured by a multi-frequency device (InBody 520) to hydrostatic weighing and reported that the device could be expected to measure fat-free mass within $12 \mathrm{lbs}$. of fat-free mass $95 \%$ of the time, which the authors deemed to be within "acceptable range". However, the proportional bias is worth noting, as the device tended to overestimate fat-free mass in the lighter wrestlers and over-estimate fat-free mass in heavier wrestlers.Conversely, our results are contrary to a previous report (Dixon, Deitrick, Pierce, Cutrufello, \& Drapeau, 2005) that a leg-to-leg BIA device underestimated the body fat \% in college wrestlers by $4.16 \%$ when compared to Bod Pod. Another study (Azcona, Koek, \& Fruhbeck, 2006) also reported that BIA underestimated $\mathrm{BF} \%$ by $3.4 \%$ and demonstrated limits of agreement spanning $13.7 \%$ in obese and non-obese children and adolescents (ages 5-22 years old) when compared to ADP. Another study (von Hurst et al., 2016) reported that the InBody 230 underestimated BF\% by an average of $2 \%$ in adults in the mid-30 year old range. Interestingly, the previously-mentioned study (Lee et al., 2017) reported a glaring $8.8 \%$ overestimation in boys and $9.7 \%$ overestimation in girls when measuring with a single-frequency device (Tanita BC418). We tested two single-frequency devices in this study (Tanita TBF-300WA plus and Accuniq BC310). The Tanita $\mathrm{BC}-418$ is a single-frequency, multi-segment analyzer similar to the specifications of the Accuniq BC 310 device used in this study, which was also the least consistent of the four devices we investigated. The Tanita TBF-300WA plus that we tested is a single-frequency, bi-polar (foot to foot) analyzer, which may explain the discrepancy. Perhaps the single frequency is inadequate for tetrapolar, multi-segmental assessments. There is no statistical significance test for the limits of agreement. As such, the acceptable limits of agreement must to be decided by the practitioner in consideration of the particular use. In this dataset, we observed large limits of agreement ranging from $15-20 \%$ (Table 6 ), and crossing zero, which means that while a device on average overestimates $\% \mathrm{BF}$, in some cases it will underestimate. These ranges can have a substantial impact on our confidence in the results from any given device. Additionally, we observed a proportional bias in two of the devices (ACC and IB270) whereby the devices showed a tendency to overestimate $\mathrm{BF} \%$ more so in those with less body fat and to underestimate $\mathrm{BF} \%$ in those with more body fat. Although these limits of agreement are objectively wider than would be preferable, they are similar to those reported in the only recent study comparing BIA to ADP (von Hurst et al., 2016). In that study of adults, the limits of agreement spanned from -4.3 to $8.4 \%$, which spans $12.7 \%$. It should be considered that there is error inherent to the device comparisons, since the referent measurement by air displacement plethysmography is itself, an estimate based on an indirect measurement of body composition. We chose the Bod Pod as the referent in this study because it is more accessible for the purposes of body composition assessment than DXA or hydrostatic weighing. For example, the California Interscholastic Federation (CIF) wrestling weight management program protocol (California Interscholastic Federation, 2016) allows for a Bod Pod assessment in the event that a wrestler disputes his or her body composition results with the approved BIA device. The most recent study investigating the validity of Bod Pod vs. DXA (Lowry \& Tomiyama, 2015) reported 95\% limits of agreement in under-, normal, and overweight/obese adults that are similar (under: 0.7-13.2\% BF; normal: -5.2$8.9 \% \mathrm{BF}$, overweight/obese: $-5.87-8.81 \% \mathrm{BF}$ ) to our BIA $95 \%$ limits of agreement which again emphasizes the amount of error that we should consider as inherent in any indirect measurement of body composition.

\section{Implications of Overestimating Body Fat}

Despite questions of the overall accuracy of these devices, when applied to the current purpose of evaluating the validity for high school wrestlers, the bias and wide limits of agreement primarily have importance for athletes at the lowest end of the body composition spectrum since the eligibility guidelines only apply to male and female wrestlers with $<7 \%$ and $<12 \%$ body fat, respectively. Particular caution should be taken when interpreting BIA (or any body composition 
test) in this group. For example, if an instrument measures a male at $8 \%$ but their true body fat percentage was $6 \%$, they would still be allowed to wrestle and even lose weight (e.g. a $125 \mathrm{lb}$. wrestler would still be permitted to lose 1.25 pounds of body fat to get down to $7 \%$ ). The initial inclination is to simply subtract the device's bias to account for the systematic (absolute) overestimation of percent body fat. However, the wide limits of agreement make this strategy problematic. To illustrate the effect of the wide limits of agreement, Table 7 shows the range of results that we could get from the different devices in the case of an individual that has $12 \%$ body fat (A), according to the Bod Pod. The "device result" (C) below is the simple result after accounting for the device's bias (B). The (D) device 95\% limits of agreement are then applied and the adjusted $95 \%$ limits of agreement shows that it is possible that an individual might actually be below the $7 \%$ body fat threshold that would exclude him from competition. Re-inspection of the Bland-Altman plots (Figure 3) shows that some individuals in this study would be in that situation. This underscores the need to pay particularly close attention to the wrestlers whose body fat is in the lower ranges, regardless of the type of body composition test that is used.

While this illustration points wide limits of agreement, the lack of precision is similar to the validation study (Utter et al., 2005) of the BIA device that is currently approved for assessing body composition in high school wrestlers in which wide ( 15 kg. (33 lb.) of fat-free mass) limits of agreement were reported as well. The researchers illustrated in their dataset, a $20 \mathrm{lb}$. underestimation of fat-free mass in one wrestler and a $26 \mathrm{lb}$. overestimation in another. The authors concluded that while the precision of the BIA device was not optimal, it was still a viable option when comparing it to the limitations associated with other body composition analysis techniques. We acknowledge limitations in our study. First, that we used a criterion method that is itself, an indirect method of body composition assessment. We chose the Bod Pod as the criterion method since some high school federation rules (e.g. California Interscholastic Federation) allow for ADP testing when the wrestler disputes their BIA results. However, while hydrostatic weighing or DXA may be considered "gold standards" of body composition assessment, they too are indirect methods of estimating body composition and are too subject to error. The second limitation of our study was that we analyzed males and females together. Due to our attempt to maximize external validity for the high school wrestling community, we only included males and females who were actively competing on their respective school teams. Because of the small proportion of female wrestlers in the community, our sample was not large enough to achieve the statistical power to perform all analyses by sex. Future work should attempt to recruit a larger sample of female wrestlers. Additionally, although recommended by BIA manufacturers, we did not exclude females who were menstruating. This was again, to increase the external validity of our results since oftentimes, wrestlers do not have the ability to choose the date that they are assessed for body composition and as such, cannot avoid testing during menses.

\section{CONCLUSIONS}

Each bioelectrical impedance device that we tested over-estimated body fat percent in high school wrestlers.

Table 6. Illustration of relationship between body fat percent on fat-free mass based on an example $150 \mathrm{lb}$. wrestler. The bias from each device was used to adjust the body fat percentage and accordingly, the fat-free mass

\begin{tabular}{lccccc}
\hline & $\begin{array}{c}\text { Example } \\
\text { Wrestler }\end{array}$ & Accuniq BC310 & InBody 120 & InBody 270 & Tanita TBF- 300WA plus \\
\hline Body mass (lbs.) & 150 & 150 & 150 & 150 & 150 \\
Body fat \% & $12.0 \%$ & $15.6 \%$ & $12.8 \%$ & $14.5 \%$ & $14.2 \%$ \\
$\begin{array}{l}\text { Fat mass (lbs.) } \\
\text { (BF\% x Body mass) }\end{array}$ & 18.0 & 23.4 & 19.2 & 21.8 & 21.3 \\
$\begin{array}{l}\text { Fat-free mass (lbs.) } \\
\text { (Body mass - Fat mass) }\end{array}$ & 132.0 & 126.6 & 130.8 & 128.2 & 128.7 \\
\hline
\end{tabular}

Table 7. Illustration of the effect of the limits of agreement on the potential range of "true" body fat percentage of a wrestler with $12 \%$ body fat, as measured by Bod Pod

\begin{tabular}{|c|c|c|c|c|c|c|}
\hline \multicolumn{7}{|l|}{ Bod Pod result: $12 \% \mathrm{BF}$} \\
\hline \multirow[t]{3}{*}{ (A) Device } & \multirow{3}{*}{$\begin{array}{l}\text { (B) } \\
\text { Bias }\end{array}$} & \multirow{3}{*}{$\begin{array}{c}\text { (C) } \\
\text { Device result }\end{array}$} & \multirow{2}{*}{\multicolumn{2}{|c|}{$\begin{array}{c}\text { (D) } \\
95 \% \text { LOA }\end{array}$}} & \multirow{2}{*}{\multicolumn{2}{|c|}{$\begin{array}{c}\text { (E) } \\
\text { Adjusted 95\% LOA }\end{array}$}} \\
\hline & & & & & & \\
\hline & & & Lower & Upper & Lower & Upper \\
\hline Accuniq BC310 & -3.63 & $15.6 \%$ & $*$ & & $9.3 \%$ & $27.8 \%$ \\
\hline InBody 120 & -0.81 & $12.8 \%$ & -8.64 & 7.03 & $5.8 \%$ & $21.4 \%$ \\
\hline InBody $270 \dagger$ & -2.53 & $14.5 \%$ & $\dagger$ & & $9.4 \%$ & $23.8 \%$ \\
\hline Tanita TBF-300WA plus & -2.20 & $14.2 \%$ & -11.31 & 6.91 & $7.3 \%$ & $25.5 \%$ \\
\hline
\end{tabular}

*95\% LOA are estimated with the regression: $-8.7-0.22 *$ mean (lower) and 10.2-0.25*mean (upper), $\uparrow 95 \%$ LOA are estimated with the regression: $-7.5-0.12 *$ mean (lower) and 6.4-0.09*mean (upper) 
Three of the devices (InBody 120, InBody 270, Tanita TBF-300WA plus) over-estimated to a similar degree $(0.8$ $2.5 \%)$, compared to air displacement plethysmography. The $4^{\text {th }}$ device (Accuniq BC310) demonstrated the least agreement of the 4 machines. It appears that the accuracy of bioelectrical impedance analysis technology is not uniform across devices and therefore, each device should be investigated before adopting for body composition assessment. The discrepancies we observed are consistent with previous work in wrestlers and reflect the impractical expectation of finding perfect agreement between two indirect methods of assessing body composition. Our results indicate that three of the devices appear to be similar in accuracy and precision to the currently approved device. Given the success of the weight management programs in preventing weight-cutting-related deaths since adopting the BIA device for body composition assessment, governing bodies for wrestling should use the research data in their decision-making process for approving appropriate devices and thus, the opportunity to implement weight management programs more broadly.

\section{REFERENCES}

Andreoli, A., Scalzo, G., Masala, S., Tarantino, U., \& Guglielmi, G. (2009). Body composition assessment by dual-energy X-ray absorptiometry (DXA). La Radiologia Medica, 114(2), 286-300. doi:10.1007/s11547-0090369-7

Armstrong, L. E., Maresh, C. M., Castellani, J. W., Bergeron, M. F., Kenefick, R. W., LaGasse, K. E., \& Riebe, D. (1994). Urinary indices of hydration status. International Journal of Sport Nutrition, 4(3), 265-279.

Azcona, C., Koek, N., \& Fruhbeck, G. (2006). Fat mass by air-displacement plethysmography and impedance in obese/non-obese children and adolescents. International Journal of Pediatric Obesity, 1(3), 176-182.

Bland, J., \& Altman, D. (1986). Statistical methods for assessing agreement between two methods of clinical measurement. Lancet, 1(8476), 307-310.

Bland, J., \& Altman, D. (1999). Measuring agreement in method comparison studies. Statistical Methods in Medical Research, 8(2), 135-160. doi:10.1177/096228029900800204

Brozek, J., Grande, F., Anderson, J., \& Keys, A. (1963). Densitometric analysis of body composition: Revision of some quantitative assumptions. Annals of the New York Academy of Sciences, 110, 113-140.

California Interscholastic Federation. (2016). 2017-2018 Wrestling weight management program manual. Retrieved from http://cifstate.org/sports/boys_wrestling/ assessor/CIF_Wrestling_Weight_Management_Handbook.pdf

Dixon, C. B., Deitrick, R. W., Pierce, J. R., Cutrufello, P. T., \& Drapeau, L. L. (2005). Evaluation of the BOD POD and leg-to-leg bioelectrical impedance analysis for estimating percent body fat in National Collegiate Athletic
Association Division III collegiate wrestlers. Journal of Strength and Conditioning Research, 19(1), 85-91. doi:10.1519/14053.1.

Fields, D. A., Goran, M. I., \& McCrory, M. A. (2002). Body-composition assessment via air-displacement plethysmography in adults and children: a review. American Journal of Clinical Nutrition, 75(3), 453-467.

Lee, L. W., Liao, Y. S., Lu, H. K., Hsiao, P. L., Chen, Y. Y., Chi, C. C., \& Hsieh, K. C. (2017). Validation of two portable bioelectrical impedance analyses for the assessment of body composition in school age children. PloS One, 12(2), e0171568. doi:10.1371/journal.pone.0171568.

Lowry, D., \& Tomiyama, A. (2015). Air displacement plethysmography versus dual-energy x-ray absorptiometry in underweight, normal-weight, and overweight/obese individuals. PloS One, 10(1), e0115086. doi:10.1371/ journal.pone.0115086.

Lozano-Nieto, A., \& Turner, A. A. (2001). Effects of orthostatic fluid shifts on bioelectrical impedance measurements.

Biomedical Instrumentation and Technology, 35(4), 249-258.

McCrory, M. A., Gomez, T. D., Bernauer, E. M., \& Mole, P. A. (1995). Evaluation of a new air displacement. plethysmograph for measuring human body composition. Medicine and Science in Sports and Exercise, 27(12), 1686-1691.

National Collegiate Athletics Association. (2016). 20162017 NCAA Wrestling Weight Management Program. Retrieved from https:/www.ncaa.org/sites/default/ files/2017DIMWR_Wrestling_Weight_Management_ Program_Information_20160929.pdf

National Federation of State High School Associations. (2016a). 2015-16 High School Athletics Participation Survey.

Retrieved from http://www.nfhs.org/ParticipationStatistics/ ParticipationStatistics.

National Federation of State High School Associations. (2016b). 2016-2017 NFHS Wrestling Rules Book.

USA Wrestling. (2016). Rule Book and Guide to Wrestling: 2017 edition. Retrieved from https://docs.google. com/document/d/1ZXYP9ZG_rFRH6hRglZMrAp8rtjn1yE1NxaOj14w0dus/edit?pli=1.

Utter, A. C., \& Lambeth, P. G. (2010). Evaluation of multifrequency bioelectrical impedance analysis in assessing body composition of wrestlers. Medicine and Science in Sports and Exercise, 42(2), 361-367. doi:10.1249/ MSS.0b013e3181b2e8b4.

Utter, A. C., Nieman, D. C., Mulford, G. J., Tobin, R., Schumm, S., McInnis, T., \& Monk, J. R. (2005). Evaluation of leg-to-leg BIA in assessing body composition of high-school wrestlers. Medicine and Science in Sports and Exercise, 37(8), 1395-1400.

von Hurst, P., Walsh, D., Conlon, C., Ingram, M., Kruger, R., \& W, S. (2016). Validity and reliability of bioelectrical impedance analysis to estimate body fat percentage against air displacement plethysmography and dual-energy X-ray absorptiometry. Nutrition and Dietetics, 73, 197-204. 